\title{
LINEAR VERIFICATION FOR SPANNING TREES
}

\author{
J. KOMLÓs
}

Received 30 Mar 1984

Given a rooted tree with values associated with the $n$ vertices and a set $A$ of directed paths (queries), we describe an algorithm which finds the maximum value of every one of the given paths, and which uses only

comparisons.

$$
5 n \div n \log \frac{|A|+n \mid}{n}
$$

This leads to a spanning tree verification algorithm using $O(n+e)$ comparisons in a graph with $n$ vertices and $c$ edges.

No implementation is oflered.

\section{Introduction}

1. Finding the minimal spanning tree in an undirected network is a wellresearched area of computer science. The classical algorithms of Kruskal and Prim have been modified and improved several times. For a study of several spanning tree algorithms, see [1].

The best known result has been the $O(|E| \log \log |V|)$ algorithm of Yao [6] until recently.

A few weeks argo. Fredman and Tarjan [2] developed a method which applies to both the shortest path and the spanning tree problems. leading to an $O(|E| \beta(|E|,|V|))$ algorithm for the latter one; where $\beta(m, n)=\min \left\{i \mid \log ^{(i)} n \equiv m / n\right\}$ On the other band, the only verification result we know of is the $O(|E| \alpha(|E|,|V|))$ algorithm of Tarjan [5]; (here $\alpha$ is the inverse Ackermann function).

Here we describe an algorithm which finds maxima over various paths of a tree, which leads to a minimal spanning tree verification algorithm with a linear number of comparisons.

We want to emphasize. however. that the only cost we deal with is the total number of comparisons made, for we could not find an effective implementation with a linear overhead cost. In other words. our result is of an information theoretica! nature. 
We remark that the problem is a particular instance of the following general question that is discussed in [3].

(Q) Given an $n$ element set $E=\left(e_{1}, \ldots, e_{n}\right)$, and a list of $m$ stibsets of $\{1,2, \ldots$ $\ldots, n\}, L=\left(S_{1}, \ldots, S_{m}\right)$. Find the maxima

$$
M_{i}=\max _{j \in s_{i}} e_{i}, \quad i=1,2, \ldots, m .
$$

(If $E$ is the set of edges of the spanning tree and the elements of $S_{i}$ are the circuit edges created by the $i$-th outside edge, we get the tree verification problem.)

of course, sorting the whole list $E$ provides all the necessary information for finding all $M_{i}$ (overhead is not counted!), but one would hope for an algorithm using only $O(m+n)$ comparisons.

Fredman proved (see [3]) that the number of possible outcomes is not more than $\left(\begin{array}{c}m+n-1 \\ n-1\end{array}\right)<2^{m+n}$, thus the above hope is realistic.

A family of paths on trees provides enough structure to make the problem easier to attack.

The general question $(Q)$ is still unanswered.

2. Given an undirected network $G$ (a graph with $n$ vertices, 2 edges and real values associated with the edges) and a spanning tree $T$ of $G$, we want to test whether $T$ is minimal among all spanning trees of $G$.

Any edge $x$ of $G$ not in $T$ creates a unique circuit $C_{x}$ with ediges of $T$; and it is well-known that $T$ is minimal if and only if, for any outside edge $x$, the value of $x$ is not smaller than any value in $C_{x}$.

Thus, we oniy need to know, for all outside $x$, the maximum value on the path $C_{x}-x$, so that we can compare this maximum with the value of $x$. Note that $C_{x}-x$ consists entirely of edges of $T$.

Let us root $T$ by a leaf of $T$. and consider it a directed tree with edges directed away from the root. Any path of $T$ is the union of at most two directed paths, and so it is sufficient to find the maxima on the directed "half-paths" corresponding to the outside edges.

By reassigning the values of the edges to their lower endpoints (and deleting the root), we get a more attractive model, in which the values are associated with the vertices.

Whatever cost we obtain for this directed path problem, we only need $2(e-n+1)$ extra comparisons for the spanning tree verification problem.

\section{Notation}

$T$ is a rooted tree with edges directed away from the root.

$V(T)=$ set of vertices of $T$

$T-U$ for a vertex set $U \subset V(T)$ is the graph $T$ restricted to $V(T)-U$

$x=y$ for $x, y \in V(T)$ means $x$ is a predecessor of $y$ on $T$

$x=y$ means $x \equiv y$ and $x \neq y$

$\operatorname{deg}(x)$ is the number of outgoing edges from $x$

path means directed path of $T$

$p(x, y)$ stands for the directed path $(u \mid x=u=y)$

$\log x$ is binary logarithm 


\section{Formulation of the problem?}

Given a rooted tree $T$ with real values associated with the vertices $f: V(T) \rightarrow \mathbf{R}$ and a set $A$ of directed paths of $T$ (queries)

$$
A \subset Q=\{p(x, y) \mid x, y \in V(T), x=y\} .
$$

Find the maximum

for all $p(x, y) \in A$.

$$
\max _{x \geqq u \cong y} f(u)
$$

We will present a solution using less than

comparisons.

$$
C=5 n+n \log \frac{|A|+n}{n}
$$

Note that $C \ll|A|$ for $|A| \gg n$, thus the linear term $O(e)$ for the tree verification problem $(|A|=e-n+1)$ comes from the comparisons made between the maxima in $A$ and the outside edges.

The paper is structured as follows. First we will describe two completely different algorithms for two particular cases: when $T$ is a string (Section 1, this is even implementable) and when $T$ is a full branching tree (Section 2), and then we will show how a general tree can be interpreted as a mixture of these two extremes.

\section{When $T$ is a string}

In other words, we have an array, $[f(i) ; 1 \leqq i \leqq n]$, and want to find maxima sver intervals $[f(i) ; s \leqq i=t]$. Although there are $\left(\begin{array}{c}n+1 \\ 2\end{array}\right)$ such intervals, we give an algorithm that uses less than $2 n$ comparisons, and still can find the answers for all $c n^{2}$ queries (with a bounded overhead per query).

This easy part of our algorithm may be folklore, but we could not trace it in the literature.

\section{Symmetric order heaps}

Given an array $[J(i) ; 1 \leqq i \equiv n]$, we construct a binary tree $S H$ on $n$ nodes with the following properries:

1. $f(i)$ is assigned to node $v_{i}$ of $S H$,

2. $S H$ is a heap; i.e. $v_{i}=v_{j}$ implies $f(i) \geqq f(j)$,

3. $v_{i}$ are in symmetric order; i.e., if $v_{j}$ belongs to the left (right) subtree of $v_{i}$ then $j<i \quad(j>i)$.

Clearly, these properties uniquely determine $S H$, namely the root of $S H$ must be $f(m)=\max _{1 \leqq i \leqq n} f(i)$, and the elements $f(i), 1 \leqq i<m(f(i), m<i \leqq n)$ should form the left subtree (right subtree) of $v_{m}$; proceed recursively inside these subtrees.

Once the tree $S H$ has been constructed, finding the maximum over subarray $[f(i) ; s \leqq i \leqq t]$ reduces to determining the lowest common ancestor of $v_{s}$ and $v_{t}$. Harel [4] has an ingenious algorithm which, after an $O(n)$ cost preprocessing, will process any number of lowest common ancestor queries for a constant cost each. 


\section{Construction of the tree $S H$}

The binary tree $S H$ will be respresented by the standard LEFTCHILD $(L C)$ and RIGHTCHILD (RC) arrays.

Definition. The right shoulder of a binary tree is the (maximal) array $S=[S(0)$, $S(1), \ldots S S(k)]$ of nodes of the tree in which $S(0)$ is the root of the tree. and $S(i)$ is the right child of $S(i-1)$.

For an easier formal description, we will use an auxiliary node $v_{0}$ with value $f(0)=+\infty$, and $S H$ will be the right subtree of $v_{0}$. Starting with this $v_{0}$, we insert the elements of $f(i)$ into the tree one-by-one, by keeping track of not only the tree structure $(L C, R C)$ but also the right shoulder array $S$. Note that $S$ forms a monotone stack $(S(k) \geqq S(k-1) \equiv \ldots \geqq S(0)$ and $f(S(k)) \leqq \ldots \leqq f(S(0)))$.

We compare the new value $f(i)$ with the stack values $f(S(j)), j=k, k-1, \ldots$, until we find an $f(S(j)) \geqq f(i)$. Then we remove the subtree $T$ with roots $S(j+1)$ from $S(j)$, add a new node $v_{i}$ (with value $f(i)$ ) to the tree as the new right child of $S(j)$, and attach the above $T$ back as the left subtree of $v_{i}$. It is clear that both the symmetric order and the heap property are preserved.

The formal description uses the arrays

$$
\begin{array}{ll}
{[f(i) ; 0 \leqq i \leqq n],} & f(0)=+\infty \\
{[L C(i), R C(i) ; 0 \leqq i \leqq n],} & L C(0)=\Lambda \\
{[S(i) ; 0 \leqq i \leqq n],} & S(0)=0 .
\end{array}
$$

Procedure SYMHEAP $(n, f, R C, L C)$

$$
\begin{aligned}
& S(0)=0 \\
& R C(0)=A \\
& k=0 \\
& \text { for } i=1 \text { to } n \text { do } \\
& j \leftarrow k \\
& \quad \text { while } f(i)>f(S(j)) \text { do } \\
& \quad j \leftarrow j-1 \\
& \quad \text { repeat } \\
& \quad k \leftarrow j+1 \\
& \quad L C(i) \leftarrow R C(S(j)) \\
& \quad R C(i) \leftarrow \Lambda \\
& R C(S(j)) \leftarrow i \\
& \quad S(j+1) \leftarrow i \\
& \text { end for } \\
& \text { end } S Y M H E A P
\end{aligned}
$$




\section{When $T$ is a full branching tree}

A rooted tree is a full branching tree, if all leaves are on the same level and non-leaves have at least 2 children. For a full binary tree, processing all path-queries has a $\operatorname{cost} \Theta(n \log \log n)$, so here we have to restrict our attention to the set $A$ of actual queries.

Let $A(y)$ be the set of actual queries which go through $y$ :

$$
A(y)=\{p(x, z) \in A \mid x \equiv y \geqq z\}
$$

and $A^{*}(y)$ the set of restrictions of these paths to the interval [ $\left.\operatorname{root}(T), y\right]$.

Starting with the root, we go down level by level and successively find the maxima over all paths in the sets $A^{*}(y)$.

Assume that we know these maxima down to the $i$-th level, and let us find the maxima on paths in $A^{*}(y)$ for a $y$ on level $i+1$. If $\bar{y}$ is the parent of $y$, then we know the maxima on the restrictions to [root, $\bar{y}$ ] of all paths in $A(y)$. It remains to compare $f(y)$ with these maxima. Since the maxima are known as an ordered list (maximum on $p(x, \bar{y})$ is at least as large as maximum on $p\left(x^{\prime}, \bar{y}\right)$ if $\left.x>x^{\prime}\right)$, we can simultaneously compare $f(y)$ with them using binary insertion. Cost $\equiv[\log (A(y)+1)]$.

Write $L_{i}$ for the set of nodes on level $i$, and $l_{i}=\left|L_{i}\right|$. For a full branching tree, $\sum_{j<i} l_{j}<l_{i}$ and thus for the entropy

$$
\sum_{i}\left(l_{i} / n\right) \log \left(n / l_{i}\right)<\sum_{i} i 2^{-i}=2 .
$$

Since $L_{i}$ is an antichain, the sets $A(y), y \in L_{i}$ are pairwise disjoint, thus Jensen's inequality leads to

$$
\sum_{y \in L_{i}}[\log (A(y)+1)]<\sum_{y \in L_{i}}(1+\log (A(y)+1)) \leqq l_{i}+l_{i} \log \frac{|A|+l_{i}}{l_{i}} .
$$

Thus, we get for the total cost:

$$
\begin{aligned}
\text { Cost } & <\sum_{i}\left(l_{i}+l_{i} \log \frac{|A|+l_{i}}{l_{i}}\right) \\
& \equiv n+n \log \frac{|A|+n}{n}+\sum l_{i} \log \frac{n}{l_{i}} \\
& \equiv 3 n+n \log \frac{|A|+n}{n} .
\end{aligned}
$$

\section{General $T$}

Scalping a rooted tree

The scalp of a tree $T$ is defined as

$$
S(T)=\{v \in V(T) \mid \operatorname{deg}(u) \equiv 1 \text { for all } u \equiv v\} .
$$

The subgraph of $T$ spanned by $S(T)$ splits into vertex-disjoint paths (of length $\geqq 0$ ) called hairs or fringes. 
We set $T_{0}=T$ and inductively define the trees $T_{i:-1}=T_{i}-S\left(T_{i}\right), i=0,1, \ldots$ and write $k$ for the index of the last non-empty $T_{i}$ (hence $S\left(T_{k}\right)=T_{k}$ ). Note that every vertex $l \in T_{i}(i=1)$ has a child in $T_{i-1}$.

For a given $i, \quad l=i: k$, and a vertex $b$ : $T_{i}$, we clefine the root of $v i n T_{i}$ as the clement

$$
R_{i}(v)=\min \left\{u \in T_{i} \mid u>v\right\} .
$$

For $v \in S\left(T_{i-1}\right)$, the "natural" root $R_{i}(v)$ will be denoted by $R^{*}(v)$. We will write $R$ for the set of all roots:

$$
R=\left\{u \mid R_{i}(v)=u \text { for some } i, v\right\} .
$$

Clearly, $R=\{u \mid$ deg $(u) \equiv 2\}$. (Note that the root of the whole tree in the traditional sense need not be in $R$.)

The restriction of $A$ (actual queries) to $T_{i}$ is defined in the natural way (by restricting the paths in $A$ to $\left.T_{i}\right)$, and is denoted by $A_{i}$. We also define $A^{*}(y)=A_{i}(y)$ for $y \in S\left(T_{i}\right)$, and $A^{+}(y)$ as the set of all queries in $A$, whose restriction to $T_{i}$ ends at $y \in S\left(T_{i}\right)$. (I.e., for $y \in S\left(T_{i}\right)$, the restrictions to $T_{i}$ of queries in $A^{+}(y)$ form the set $A^{*}(y)$.) (The rest of the section is not needed for the description of the algorithm, only for the cost analysis.) In other words, a query $p(x, z) \in A^{+}(y)$ (where $y \in S\left(T_{i}\right)$ ) iff $R_{i}(z)=y$. But then $R(v)=y$ for all $v, y \geqq v \geqq z$. Hence, if $y_{1} \in S\left(T_{i}\right), y_{2} \in S\left(T_{j}\right)$, $i>j$, and a query $p(x, z)$ belongs to both $A^{+}\left(y_{1}\right)$ and $A^{+}\left(y_{2}\right)$, then $R_{i}\left(y_{2}\right)=y_{1}$.

Thus, we obtained the following lemma.

Lemma 1. If $Y$ is a set of vertices such that no elentent in $Y$ is a root of another, then the sets $A^{+}(y), y \in Y$, are pairwise disjoint.

For an element $u \in S\left(T_{i}\right)$, the set $C(u)=\left\{v \mid R_{i}(v)=u\right\}$ is called the court of $u$. Now we are going to partition $R$ by coloring its elements with $k$ colors. The elements of $S\left(T_{1}\right) \cap R$ get color 1 . Having colored the elements of

$$
\bigcup_{I \geqq j=i}\left[S\left(T_{j}\right) \cap R\right]
$$

we color the elements of $S\left(T_{i}\right) \cap R$ as follows: $u$ gets the smallest color (smallest positive integer) that does not appear in its court $C(u)$. Clearly, in the obtained coloring an element in $S\left(T_{i}\right) \cap R$ gets a color not exceeding $i$. We will write $R_{i}$ for the set of $u \in R$ with color $i, R_{0}$ for the set of leaves of $T_{0}, r_{i}$ for $\left|R_{i}\right|$, and $r$ for $|R|$.

The following crucial lemma will be proved at the end of the paper.

Lemma 2. A) For any fixed $i=1$, the sets $A^{+}(y), y \in R_{i}$, are disjoint.

B) For $i \geqq 0$, we have the exponential decay

whence $r<n / 2$, and

$$
\sum_{i=i} r_{i}<r_{i}
$$

$$
\sum_{j \rightarrow i} i_{i} \cdot r 2^{-i}, \quad i=1,2, \ldots
$$

consequently, for the entropy

$$
\sum_{i \cong 1}\left(r_{i} / r\right) \log \left(r / r_{i}\right)<\sum_{i \lesssim 1} i 2^{-i}=2 .
$$

In other words, the coloring defined above provides a finite-entropy decomposition of $R$ into antichains in the partial order $u \gg v$ if $u=R_{i}(v)$ for some $i$. 


\section{The Algorithm}

We start with the decomposition

$$
V(T)=\sum_{i=0}^{k} S\left(T_{i}\right) \quad \text { (disjoint union) }
$$

Since every scalp $S\left(T_{i}\right)$ represents a disjoint union of paths, $V(T)$ is decomposed into disjoint sets, each of which spans a (directed) path of $T$.

Perform SYMHEAP on each of these sets. Total cost is less than $2 n$. Next, starting with $A_{k}$, we will inductively find the answers for all queries in $A_{i}, i=k$, $k-1, \ldots, 0\left(A_{0}=A\right.$ is the original set of queries).

For $i=k$, the tree $T_{k}$ is but a string, and SYMHEAP provided us with the maximum on all possible paths of $T_{k}$.

Having answered all queries in $A_{i+1}$, we proceed to get the answers for queries in $A_{i}-A_{i+1}$. For a given vertex $y \in S\left(T_{i}\right)$ there are $\left|A_{i}(y)\right|$ queries in $A_{i}$ that end at $y$. For a particular query $p(x, y) \in A_{i}$ we already know an index $m_{x y}$ such that

$$
f\left(m_{x y}\right)=\max _{x \equiv u \geqq R^{*}(y)} f(u)
$$

(for $x<R^{i}(y)$ we may interpret $f\left(m_{x y}\right)=0$ ), and from SYMHEAP, we know an index $m_{y}$ such that

$$
f\left(m_{y}\right)=\max _{R^{*}(y)>u \geq y} f(u) .
$$

It remains to compare $f\left(m_{y}\right)$ with $f\left(m_{x y}\right)$. For fixed $y$, we know the order of the values $f\left(m_{x y}\right)$, since $x_{1}>x_{2}$ implies $f\left(m_{x, y}\right) \triangleq f\left(m_{x_{2 y}}\right)$. Thus, we can make the comparisons of $f\left(m_{y}\right)$ with the various values $f\left(m_{x y}\right)$ simultaneously, by merging the value $f\left(m_{y}\right)$ into the ordered sequence $f\left(m_{x_{1} y}\right) \geqq f\left(m_{x_{2 y} y}\right) \ldots$ Cost is at most $\left\lceil\log \left(\left|A_{i}(y)\right|+1\right)\right\rceil=$ $=\left\lceil\log \left(\left|A^{*}(y)\right|+1\right)\right\rceil$ for every $y \in S\left(T_{i}\right)$.

\section{Cost Analysis}

Theorem. The obtained total cost

is less than

$$
C=2 n+\sum_{y}\left[\log \left(\left|A^{*}(y)\right|+1\right)\right]
$$

$$
5 n+n \log \frac{|A|+n}{n} .
$$

Proof. We will separately handle terms with $y \in R$ and those with $y \in R$.

By Lemma 1, the sets $A^{+}(y), y \in R$, are pairwise disjoint, thus applying the Jensen inequality

$$
\sum_{i=1}^{t} \log x_{i} \triangleq i \log \frac{\sum x_{i}}{t}
$$

we get

$$
\sum_{y \in R}\left[\log \left(\left|A^{*}(y)\right|+1\right)\right] \approx \sum\left[1+\log \left(\left|A^{*}(y)\right|+1\right)\right] \leqq m+m \log \frac{|A|+m}{m}
$$

where $m=n-r$ is the number of non-roots. 
It remains to estimate the sum

$$
C^{1}=\sum_{i=1}^{k} \sum_{y \in R_{i}}\left\lceil\log \left(\mid A^{*}\left(y^{\prime}\right)+1\right) \mid\right. \text {. }
$$

Here we may deal with different restrictions of the same path, so multiplicities may occur. By part $A$ of Lemma 2, however, we can use the above estimation for the inner sums:

$$
C^{1}=\sum_{i=1}^{k}\left[r_{i}+r_{i} \log \frac{|A|+r_{i}}{r_{i}}\right] \text {. }
$$

The exponential decay of the sizes $r_{i}$ does the rest (part $B$ of Lemmal 2):

$$
C^{1}<r+r \log \frac{|A|+r}{r}+\sum_{i=1} r_{i} \log \frac{r}{r_{i}}=3 r+r \log \frac{|A|+r}{r} .
$$

Now $n+r=n$, thus. (using $r<n / 2$ and the log-sum inequality

$$
\left.x \log \frac{a}{x}+y \log \frac{b}{y}=(x+y) \log \frac{a+b}{x+y}, x, y>0\right)
$$

we get for the total cost

as stated.

$$
C=2 n+m+3 r+m \log \frac{|A|+m}{n}+r \log \frac{|A|+r}{r}<5 n+n \log \frac{|A|+n}{n}
$$

Proof of Lemma 2. The sets $R$ are clearly antichains of $T$ in the partial order $\gg$, so part $A$ follows from Lemma 1 . To establish the exponential decay of $r_{i}=\left|R_{i}\right|$, we need two more lemmas:

Lemma 3. Leaves of $T_{i}$ ( $i \geqq 1$ ) have color $i$. Furthermore, for $i \geqq 2$ and $u \in R_{i}$, the court $C(u)$ contains exactly the colors $\{1, \ldots, i-1\}$.

Lemma 4. Every node $u \in \bigcup_{j<i} R_{j}$ must have at least two children in $T_{i}$.

To get the exponential decay, it remains to apply the following simple fact: In any rooted tree, the number of vertices of degree $\geq 2$ is less than the number of leaves.

Proof of Lemma 3. For $i=1$ the statement is trivial. Assuming its validity for all values less than a certain $i$, let us prove it for $i$.

We know that a leaf $u$ of $T_{i}$ (actually any vertex of $T_{i}$ ) has a child in $T_{i-1}$, thus there is a leaf $v$ of $T_{i-1}$ such that $R_{i}(v)=u$. Since (by induction) the color of $v$ is $i-1$, and $C(v)$ (a subset of $C(u)$ ) contains all colors $\{1,2, \ldots, i-2\}, u$ must have color $=i$. But $u \in S\left(T_{i}\right)$, so its color cannot exceed $i$, thus it is $i$.

Furthermore, any vertex $u \in R_{i}$ has (by definition) a vertex $\imath \in R_{i-1}$ in its court $C(u)$. By induction, $C(v)$ contains all colors $\{1, \ldots, i-2\}$, thus the relation $C(v) \subset C(u)$ implies the second statement of the lemma.

Proof of Lemma 4. If $u \in \bigcup_{j=i} R_{j}$, then $u$ has a vertex $v \in R_{i}$ in its court $C(u)$. $R_{i} \subseteq T_{i}$ implies $v \in T_{i}$. Restricting oursclves to the tree $T_{i}$, we found a vertex $v$ to which $u$ is a root. Thus, the degree of $u$ within $T_{i}$ must be at least 2 . 


\section{References}

[1] D. CherIton and R. E. TaRJAN, Finding Minimum Spanning Trees, SIAM J. on Computing, 5 (1976), $724-742$.

[2] M. FREDMAN and R. E. TARJAN, private communication, December 1983.

[3] R. L. Grahim, A. C. YAO, and F. F. Yao, Information Bounds are Weak in the Shortest Distance Problem, JACM, 27 (1980), 428- 444.

[4] D. HAREL, A Linear Time Algorithm for the Lowest Common Ancestors Problem, Proc. 21st Annual Symp. on Foundations of Computer Science, (1980), 308-319.

[5] R. E. TARJAN, Application of Path Compression on Balanced Trees, $J A C M, 26(1979), 690-715$.

[6] A. C. YAo, An $O(|E| \log \log |V|)$ Algorithm for Finding Minimum Spanning Trees, Information Processing Letters, 4 (1975), 21-23.

\section{J. Komlós}

Mathematical Institute of the

Hungarian Acadenty of Scientes.s

Budupest, P.O.B. 127

1364, Hungasy

and

University of California. San Diego

La Jolla, CA 92093, U.S.A. 